\title{
DECOMPOSITION OF DATA AND VARIATION IN THE ANALYTIC HIERARCHY PROCESS
}

\author{
Shin'ya Nagasawa \\ College of Business Administration, Ritsumeikan University \\ 1-1-1 Noji-kita, Kusatsu, Shiga 525-8577 Japan \\ snt01497@ba.ritsumei.ac.jp
}

\begin{abstract}
In order to improve Analytic Hierarchy Process (AHP), this paper proposes the decomposition of data in the AHP by the logarithmic linear model. Data are decomposed to a main effect, an order effect, a combination effect, personal interactions and errors by the proposed mode. The proposed model is considered to be useful to analyze details of decision making by the AHP.
\end{abstract}

In order to improve Saaty's Analytic Hierarchy Process (AHP) this paper proposes the decomposition of data and variation in the AHP by the following logarithmic linear model;

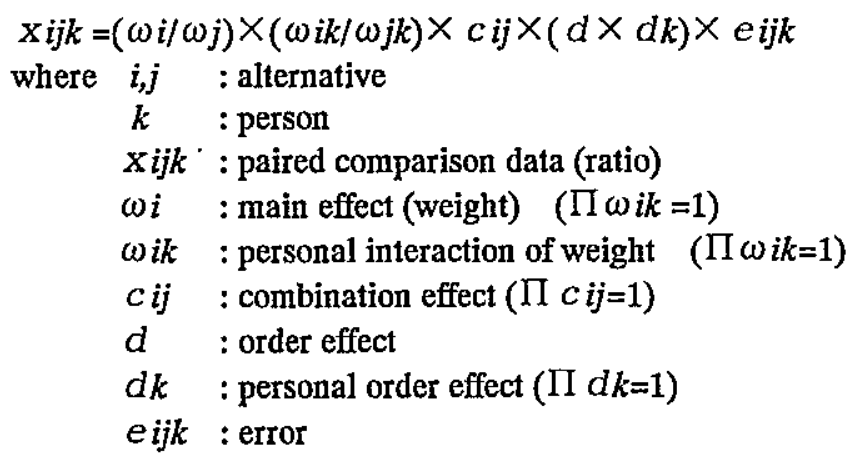

By logarithmic least squares method, data and variation are decomposed to a main effect, an order effect, a combination effect, personal interactions and errors by the proposed mode. The proposed model is considered to be useful to analyze details of decision making by the AHP.

Table 1 Inverse order AHP data by 3 persons

\begin{tabular}{cccccc}
\hline$i \backslash j$ & & 1 & 2 & 3 & 4 \\
\hline \multirow{4}{*}{1} & $\mathrm{P}_{1}$ & & $2^{0}$ & $2^{-1}$ & $2^{-1}$ \\
& $\mathrm{P}_{2}$ & 1 & $2^{0}$ & $2^{-2}$ & $2^{-2}$ \\
& $\mathrm{P}_{3}$ & & $2^{1}$ & $2^{-1}$ & $2^{-1}$ \\
& $\mathrm{P}_{1}$ & $2^{3}$ & & $2^{0}$ & $2^{0}$ \\
2 & $\mathrm{P}_{2}$ & $2^{0}$ & 1 & $2^{-2}$ & $2^{-2}$ \\
& $\mathrm{P}_{3}$ & $2^{1}$ & & $2^{0}$ & $2^{-2}$ \\
& $\mathrm{P}_{1}$ & $2^{3}$ & $2^{1}$ & & $2^{-1}$ \\
3 & $\mathrm{P}_{2}$ & $2^{3}$ & $2^{1}$ & 1 & $2^{-1}$ \\
& $\mathrm{P}_{3}$ & $2^{2}$ & $2^{1}$ & & $2^{-1}$ \\
& $\mathrm{P}_{1}$ & $2^{3}$ & $2^{3}$ & $2^{1}$ & \\
4 & $\mathrm{P}_{2}$ & $2^{3}$ & $2^{3}$ & $2^{1}$ & 1 \\
& $\mathrm{P}_{3}$ & $2^{2}$ & $2^{1}$ & $2^{0}$ & \\
\hline
\end{tabular}

Table 2 Analysis of geometric mean data (1)

\begin{tabular}{cccccc}
\hline$i \backslash j$ & 1 & 2 & 3 & 4 & weight \\
\hline 1 & 1 & $2^{-0.50}$ & $2^{-2.00}$ & $2^{-2.00}$ & 0.095 \\
2 & & 1 & $2^{-0.83}$ & $2^{0.1 .83}$ & 0.143 \\
3 & & & 1 & $2^{0-0.83}$ & 0.294 \\
4 & & & & 1 & 0.467 \\
\hline & & & \\
C.I. $=0.013$, C.R. $=0.014$
\end{tabular}

Table 3 Analysis of geometric mean data (2)

\begin{tabular}{cccccc}
\hline$i \backslash j$ & 1 & 2 & 3 & 4 & weight \\
\hline 1 & 1 & $2^{0.33}$ & $2^{-1.33}$ & $2^{-1.33}$ & 0.095 \\
2 & $2^{1.33}$ & 1 & $2^{-0.67}$ & $2^{-1.33}$ & 0.143 \\
3 & $2^{2.67}$ & $2^{1.00}$ & 1 & $2^{-1.00}$ & 0.294 \\
4 & $2^{2.67}$ & $2^{2.33}$ & $2^{0.67}$ & 1 & 0.467 \\
\hline & & & C.I. $=0.430$, C.R. $=0.477$
\end{tabular}


Table 4 Decomposition of Data in AHP

(a) ratio of main effect (weight) $\omega i / \omega j$

\begin{tabular}{|c|c|c|c|c|c|c|}
\hline$i \backslash j$ & & 1 & 2 & 3 & 4 & weight \\
\hline & $P_{1}$ & & $2^{-0.58}$ & $2^{-1.62}$ & $2^{-2.29}$ & \\
\hline \multirow[t]{3}{*}{1} & $\mathrm{P}_{2}$ & 1 & $2^{-0.58}$ & $2^{-1.62}$ & $2^{-2.29}$ & 0.095 \\
\hline & $\mathrm{P}_{3}$ & & $2^{-0.58}$ & $2^{-1.62}$ & $2^{-2.29}$ & \\
\hline & $\mathrm{P}_{1}$ & $2^{0.58}$ & & $2^{-1.04}$ & $2^{-1.71}$ & \\
\hline \multirow[t]{3}{*}{2} & $\mathrm{P}_{2}$ & $2^{0.58}$ & 1 & $2^{-1.04}$ & $2^{-1.71}$ & 0.143 \\
\hline & $\mathrm{P}_{3}$ & $2^{0.58}$ & & $2^{-1.04}$ & $2^{\cdot 1.71}$ & \\
\hline & $P_{1}$ & $2^{1.62}$ & $2^{1.04}$ & & $2^{-0.67}$ & \\
\hline \multirow[t]{3}{*}{3} & $\mathrm{P}_{2}$ & $2^{1.62}$ & $2^{1.04}$ & 1 & $2^{-0.67}$ & 0.294 \\
\hline & $P_{3}$ & $2^{1.62}$ & $2^{1.04}$ & & $2^{-0.67}$ & \\
\hline & $P_{1}$ & $2^{2.29}$ & $2^{1.71}$ & $2^{0.67}$ & & \\
\hline \multirow[t]{2}{*}{4} & $\mathrm{P}_{2}$ & $2^{2.29}$ & $2^{1.71}$ & $2^{0.67}$ & 1 & 0.467 \\
\hline & $\mathrm{P}_{3}$ & $2^{2.29}$ & $2^{1.71}$ & $2^{0.67}$ & & \\
\hline
\end{tabular}

(b) ratio of personal interaction $\omega i k / \omega j k$

\begin{tabular}{ccccccc}
\hline$i \backslash j$ & & 1 & 2 & 3 & 4 & weight \\
\hline \multirow{4}{*}{1} & $\mathrm{P}_{1}$ & & $2^{0.67}$ & $2^{-0.12}$ & $2^{-0.21}$ & \\
& $\mathrm{P}_{2}$ & 1 & $2^{0.33}$ & $2^{-0.38}$ & $2^{-0.46}$ & 0.25 \\
& $\mathrm{P}_{3}$ & & $2^{0.33}$ & $2^{0.50}$ & $2^{0.67}$ & \\
& $\mathrm{P}_{1}$ & $2^{0.67}$ & & $2^{0.54}$ & $2^{0.46}$ & \\
2 & $\mathrm{P}_{2}$ & $2^{-0.33}$ & 1 & $2^{-0.71}$ & $2^{-0.79}$ & 0.25 \\
& $\mathrm{P}_{3}$ & $2^{-0.33}$ & & $2^{0.17}$ & $2^{0.33}$ & \\
& $\mathrm{P}_{1}$ & $2^{0.12}$ & $2^{-0.54}$ & & $2^{-0.08}$ & \\
3 & $\mathrm{P}_{2}$ & $2^{0.38}$ & $2^{0.71}$ & 1 & $2^{-0.08}$ & 0.25 \\
& $\mathrm{P}_{3}$ & $2^{-0.50}$ & $2^{-0.17}$ & & $2^{0.17}$ & \\
& $\mathrm{P}_{1}$ & $2^{0.21}$ & $2^{-0.46}$ & $2^{0.08}$ & & \\
4 & $\mathrm{P}_{2}$ & $2^{0.46}$ & $2^{0.79}$ & $2^{0.08}$ & 1 & 0.25 \\
& $\mathrm{P}_{3}$ & $2^{-0.67}$ & $2^{-0.33}$ & $2^{-0.17}$ & \\
\hline & & \multicolumn{5}{c}{ C.I. $=0$, C.R. $=0$} \\
\end{tabular}

(c) combination effect $c i$

\begin{tabular}{ccccccc}
\hline$i \backslash j$ & & 1 & 2 & 3 & 4 & weight \\
\hline & $\mathrm{P}_{1}$ & & $2^{0.08}$ & $2^{-0.38}$ & $2^{0.29}$ & \\
1 & $\mathrm{P}_{2}$ & 1 & $2^{0.08}$ & $2^{-0.38}$ & $2^{0.29}$ & 0.25 \\
& $\mathrm{P}_{3}$ & & $2^{0.08}$ & $2^{-0.38}$ & $2^{0.29}$ & \\
& $\mathrm{P}_{1}$ & $2^{-0.08}$ & & $2^{0.21}$ & $2^{-0.12}$ & \\
2 & $\mathrm{P}_{2}$ & $2^{-0.08}$ & 1 & $2^{0.21}$ & $2^{-0.12}$ & 0.25 \\
& $\mathrm{P}_{3}$ & $2^{-0.08}$ & & $2^{0.21}$ & $2^{-0.12}$ & \\
& $\mathrm{P}_{1}$ & $2^{0.38}$ & $2^{-0.21}$ & & $2^{-0.17}$ & \\
3 & $\mathrm{P}_{2}$ & $2^{0.38}$ & $2^{-0.21}$ & 1 & $2^{-0.17}$ & 0.25 \\
& $\mathrm{P}_{3}$ & $2^{0.38}$ & $2^{-0.21}$ & & $2^{-0.17}$ & \\
& $\mathrm{P}_{1}$ & $2^{-0.29}$ & $2^{0.12}$ & $2^{0.17}$ & & \\
4 & $\mathrm{P}_{2}$ & $2^{-0.29}$ & $2^{0.12}$ & $2^{0.17}$ & $1^{2}$ & 0.25 \\
& $\mathrm{P}_{3}$ & $2^{-0.29}$ & $2^{0.12}$ & $2^{0.17}$ & \\
\hline \multicolumn{6}{c}{ C.I. $=0.013$, C.R. $=0.014$}
\end{tabular}

(d) order effect $d$

\begin{tabular}{ccccccc}
\hline$i \backslash j$ & & 1 & 2 & 3 & 4 & weight \\
\hline \multirow{4}{*}{1} & $\mathrm{P}_{1}$ & & $2^{0.44}$ & $2^{0.44}$ & $2^{0.44}$ & \\
& $\mathrm{P}_{2}$ & 1 & $2^{0.44}$ & $2^{0.44}$ & $2^{0.44}$ & 0.25 \\
& $\mathrm{P}_{3}$ & & $2^{0.44}$ & $2^{0.44}$ & $2^{0.44}$ & \\
& $\mathrm{P}_{1}$ & $2^{0.44}$ & & $2^{0.44}$ & $2^{0.44}$ & \\
2 & $\mathrm{P}_{2}$ & $2^{0.44}$ & 1 & $2^{0.44}$ & $2^{0.44}$ & 0.25 \\
& $\mathrm{P}_{3}$ & $2^{0.44}$ & & $2^{0.44}$ & $2^{0.44}$ & \\
& $\mathrm{P}_{1}$ & $2^{0.44}$ & $2^{0.44}$ & & $2^{0.44}$ & \\
3 & $\mathrm{P}_{2}$ & $2^{0.44}$ & $2^{0.44}$ & 1 & $2^{0.44}$ & 0.25 \\
& $\mathrm{P}_{3}$ & $2^{0.44}$ & $2^{0.44}$ & & $2^{0.44}$ & \\
& $\mathrm{P}_{1}$ & $2^{0.44}$ & $2^{0.44}$ & $2^{0.44}$ & & \\
4 & $\mathrm{P}_{2}$ & $2^{0.44}$ & $2^{0.44}$ & $2^{0.44}$ & 1 & 0.25 \\
& $\mathrm{P}_{3}$ & $2^{0.44}$ & $2^{0.44}$ & $2^{0.44}$ & \\
\hline \multicolumn{6}{c}{ C.I. $=0.360$, C.R. $=0.400$}
\end{tabular}

(e) personal order effect $d k$

\begin{tabular}{|c|c|c|c|c|c|c|}
\hline$i \backslash j$ & & 1 & 2 & 3 & 4 & weight \\
\hline \multirow{4}{*}{1} & $P_{1}$ & & $2^{0.47}$ & $2^{0.47}$ & $2^{0.47}$ & \\
\hline & $\mathrm{P}_{2}$ & 1 & $2^{-0.28}$ & $2^{-0.28}$ & $2^{.0 .28}$ & 0.25 \\
\hline & $\mathrm{P}_{3}$ & & $2^{-0.19}$ & $2^{-0.19}$ & $2^{-0.19}$ & \\
\hline & $P_{1}$ & $2^{0.47}$ & & $2^{0.47}$ & $2^{0.47}$ & \\
\hline \multirow[t]{3}{*}{2} & $\mathrm{P}_{2}$ & $2^{-0.28}$ & 1 & $2^{-0.28}$ & $2^{-0.28}$ & 0.25 \\
\hline & $\mathrm{P}_{3}$ & $2^{-0.19}$ & & $2^{-0.19}$ & $2^{-0.19}$ & \\
\hline & $P_{1}$ & $2^{0.47}$ & $2^{0.47}$ & & $2^{0.47}$ & \\
\hline \multirow[t]{3}{*}{3} & $\mathrm{P}_{2}$ & $2^{-0.28}$ & $2^{-0.28}$ & 1 & $2^{-0.28}$ & 0.25 \\
\hline & $\mathrm{P}_{3}$ & $2^{-0.19}$ & $2^{-0.19}$ & & $2^{-0.19}$ & \\
\hline & $P_{1}$ & $2^{0.47}$ & $2^{0.47}$ & $2^{0.47}$ & & \\
\hline \multirow[t]{2}{*}{4} & $\mathrm{P}_{2}$ & $2^{-0.28}$ & $2^{-0.28}$ & $2^{-0.28}$ & 1 & 0.25 \\
\hline & $\mathrm{P}_{3}$ & $2^{-0.19}$ & $2^{-0.19}$ & $2^{-0.19}$ & & \\
\hline
\end{tabular}

Nagasawa, S.(1993) Pcoceedings of the Autumn Conference of ORSJ, pp.220-221.(in Japanese) 